\title{
Proposal for a local heating driven spin current generator
}

\author{
Sun-Yong Hwang, ${ }^{1,2}$ Jong Soo Lim, ${ }^{2,3}$ Rosa López, ${ }^{2,4}$ Minchul Lee, ${ }^{5}$ and David Sánchez ${ }^{2,4}$ \\ ${ }^{1}$ Department of Physics, Pohang University of Science and Technology, Pohang 790-784, Korea \\ ${ }^{2}$ Institut de Física Interdisciplinària i Sistemes Complexos IFISC (UIB-CSIC), E-07122 Palma de Mallorca, \\ Spain \\ ${ }^{3}$ School of Physics, Korea Institute for Advanced Study, Seoul 130-722, Korea \\ ${ }^{4}$ Departament de Física, Universitat de les Illes Balears, E-07122 Palma de Mallorca, Spain \\ ${ }^{5}$ Department of Applied Physics, College of Applied Science, Kyung Hee University, Yongin 446-701, Korea
}

(Received 29 August 2013; accepted 3 October 2013; published online 21 October 2013)

\begin{abstract}
We propose a two-terminal spin-orbit interferometer with a hot molecule inserted in one of its arms to generate pure spin currents. Local heating is achieved by coupling the vibrational modes of the molecule to a third (phononic) reservoir. We show that this spin caloritronic effect is due to the combined influence of spin-dependent wave interference and inelastic scattering. Remarkably, the device converts heat flow into spin-polarized current even without applying any voltage or temperature difference to the electronic terminals. (C) 2013 AIP Publishing LLC. [http://dx.doi.org/10.1063/1.4826108]
\end{abstract}

Recent experimental demonstrations of spin-polarized currents using thermal gradients only ${ }^{1,2}$ have fueled the interest in finding synergies between thermoelectricity and spintronics. Thus, the field of spin caloritronics ${ }^{3}$ seeks new functionalities that exploit the coupling of charge, spin, and energy degrees of freedom in nanostructures. Here we propose a molecule-based spin caloritronic device that extracts heat from a nearby phonon bath and transforms it into a spin current that flows out into coupled electronic reservoirs. Crucial to our setup is the presence of tunable spin-orbit interactions, which causes traveling electrons to acquire a phase that depends on its spin orientation within an Aharonov-Bohm-type interferometer.

Recent works predict that heat current can be converted into electric current in three-terminal thermoelectric nanodevices either due to inelastic processes at a molecular bridge ${ }^{4}$ or due to Coulomb coupling in interacting quantum dots. ${ }^{5}$ The generated charge current is determined by the temperature difference between the third terminal kept at an elevated temperature and the base temperature of the system. Further investigations include time-reversal symmetry breaking effects, ${ }^{6}$ chaotic cavity heat engines, ${ }^{7}$ and phonon-assisted instabilities. ${ }^{8}$ The effect originates from a rectification of temperature fluctuations in the coupled unbiased conductor, similarly to the directed motion induced in the drag effect. ${ }^{9}$ Importantly, unlike the pure electric case which requires four current-carrying terminals, in these thermoelectric effects it suffices to couple a third energy-supplying subsystem (electronic or phononic).

Our proposal considers an Aharonov-Bohm (AB) ring connected to two normal electronic reservoirs and a molecule embedded in one arm of the ring, see Fig. 1. For definiteness, the molecule is assumed to have a single level active for transport. The system is subjected to Rashba spinorbit interaction arising in inversion asymmetric potentials. This configuration has been shown to give rise to local spin polarizations, ${ }^{10}$ spin separation, ${ }^{11}$ and pure spin currents. ${ }^{12}$ Furthermore, this is a useful setup to analyze the competition between spin randomizing Rashba interactions and manybody spin singlets arising from Kondo correlations. ${ }^{13,14}$ These works require small voltage biases applied to the
Fermi reservoirs. In contrast, our device operates in the entire absence of electric or thermal gradients applied to the leads, but is instead based on a hot phonon field locally coupled to the embedded molecule.

The model Hamiltonian for our device, $\mathcal{H}=\mathcal{H}_{C}+\mathcal{H}_{M}$ $+\mathcal{H}_{T}$, consists of three parts: $\mathcal{H}_{C}=\sum_{\ell=L / R, k, \sigma} \varepsilon_{k \sigma} c_{\ell k \sigma}^{\dagger} c_{\ell k \sigma}$ is the Hamiltonian of two normal left $(L)$ and right $(R)$ electronic reservoirs, $\mathcal{H}_{M}=\sum_{\sigma} E_{d} d_{\sigma}^{\dagger} d_{\sigma}+\hbar \omega_{0} a^{\dagger} a+\lambda\left(a+a^{\dagger}\right)$ $\sum_{\sigma} d_{\sigma}^{\dagger} d_{\sigma}$ describes the embedded molecule with level $E_{d}$ coupled to a phonon bath with an excitation frequency $\omega_{0}$ and coupling strength $\lambda$, and $\mathcal{H}_{T}=\sum_{\ell, k, \sigma}\left(V_{\ell} c_{\ell k \sigma}^{\dagger} d_{\sigma}+\right.$ H.c. $)$ $+\sum_{k, p, \sigma}\left(W e^{i \varphi_{\sigma}} c_{R p \sigma}^{\dagger} c_{L k \sigma}+\right.$ H.c. $)$ accounts for electron tunneling between the molecule and the reservoirs. Here, $c_{\ell k \sigma}\left(c_{\ell k \sigma}^{\dagger}\right)$ are fermionic annihilation (creation) operators for electrons with wave vector $k$ and spin $\sigma$ in lead $\ell=L / R$ and $d_{\sigma}\left(d_{\sigma}^{\dagger}\right)$ represent electrons at the molecule site and $a\left(a^{\dagger}\right)$ denotes the bosonic annihilation (creation) operator. $V_{\ell}$ is the probability amplitude for an electron transfer between the molecule and the electronic reservoirs whereas $W$ describes direct tunneling between electronic reservoirs. The effect of Rashba spinorbit interaction acting upon the molecule is embodied in the phase factor ${ }^{10-14} \varphi_{\sigma}=\sigma \varphi$, where $\varphi=\alpha_{R} l\left(\alpha_{R}\right.$ is the Rashba strength and $l$ the size of the molecule) and $\sigma=+(-)$ for spins up (down).

We consider thermoelectric effects in the linear response regime. Thus, we expand the electrochemical potential and temperature in each electronic reservoir around the equilibrium state defined with common chemical potential $\mu$ and base temperature $T$. Then, $\mu_{\ell \sigma}=\mu+\Delta \mu_{\ell \sigma}$ and $T_{L / R}=T \pm \Delta T / 2$; similarly, $T_{P}=T+\Delta T_{P}$ for the phonon bath. Using $\Delta \mu_{\sigma}$ $=\mu_{L \sigma}-\mu_{R \sigma}$, we define the charge and spin voltage biases $e \Delta V=\left(\Delta \mu_{\uparrow}+\Delta \mu_{\downarrow}\right) / 2$ and $e \Delta V_{s}=\left(\Delta \mu_{\uparrow}-\Delta \mu_{\downarrow}\right) / 2$, respectively. ${ }^{15}$ Hence, the spin-resolved current is

$$
I_{\sigma}=G\left(\varphi_{\sigma}\right) \Delta V+\sigma G\left(\varphi_{\sigma}\right) \Delta V_{s}+L\left(\varphi_{\sigma}\right) \frac{\Delta T}{T}+X_{P}\left(\varphi_{\sigma}\right) \frac{\Delta T_{P}}{T} .
$$

The transport coefficient $G$ is the linear conductance in response to a charge or spin voltage shift. $L$ is the thermoelectric response due to a temperature difference applied to 


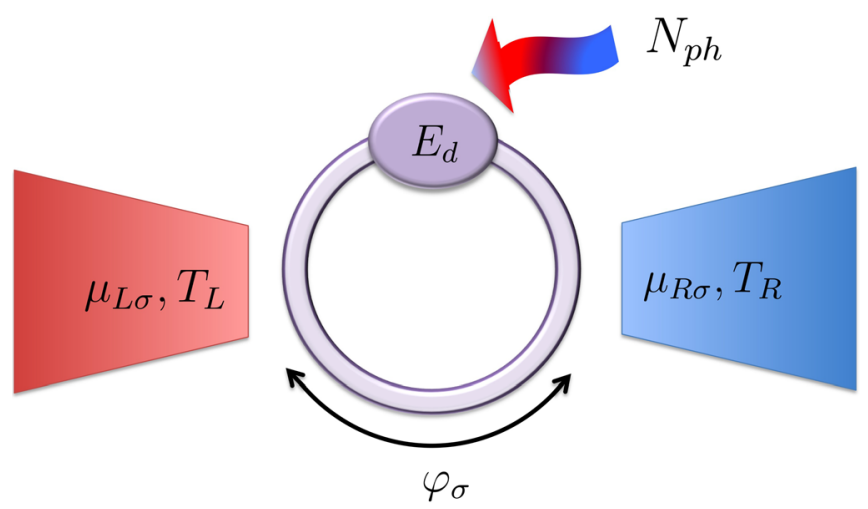

FIG. 1. Schematics of the setup. A molecule or quantum dot with a single level $E_{d}$ is embedded in an Aharonov-Bohm ring coupled to two Fermi reservoirs $\left(L\right.$ and $R$ ) with electrochemical potentials $\mu_{L(R)}$ and temperatures $T_{L(R)}$. A spin-dependent phase $\varphi_{\sigma}$ originates from spin-orbit Rashba interactions, generally leading to spin-dependent chemical potentials, $\mu_{\ell \sigma}$. Our device is influenced by the presence of a bosonic bath described by the Bose-Einstein distribution function $N_{p h}$ with temperature $T_{P}$.

the electronic leads. Finally, $X_{P}$ describes phonon-assisted transport owing to possible temperature differences between the bosonic bath and the system. We remark that local phonon heating effects have been investigated in electronic molecular systems. ${ }^{16,17}$

Importantly, the transport coefficients in Eq. (1) have elastic and inelastic contributions: $G=G_{e l}+G_{P}$ and $L=L_{e l}$ $+L_{P}$. Expressions for $G_{e l}$ and $L_{e l}$ can be found in Ref. 6 with the replacement $\varphi_{A B} \rightarrow \varphi_{\sigma}$. We here prefer to focus on the inelastic contribution originated from electron-phonon (e-ph) coupling since it is precisely the combined effect of inelastic scattering and spin-orbit interaction which establishes the operating principle of our proposed device. Within the nonequilibrium Green function approach, ${ }^{18}$ we find $G_{P}\left(\varphi_{\sigma}\right)=$ $\left(2 \lambda^{2} e^{2} / h\right) \int d \varepsilon B\left(\varepsilon, \varphi_{\sigma}\right) M_{0}\left(\varepsilon, \varphi_{\sigma}\right)$, where $B\left(\varepsilon, \varphi_{\sigma}\right)=\left(N_{T} / k_{B} T\right)$ $\tilde{\Gamma}\left(\varepsilon_{+}\right) \tilde{\Gamma}\left(\varepsilon_{-}\right)\left|\mathscr{G}_{\sigma, \sigma}^{r}\left(\varepsilon_{+}\right) \mathscr{G}_{\sigma, \sigma}^{r}\left(\varepsilon_{-}\right)\right|^{2}\left(1-f_{0}\left(\varepsilon_{+}\right)\right) f_{0}\left(\varepsilon_{-}\right) \quad$ and $M_{0}\left(\varepsilon, \varphi_{\sigma}\right) \equiv 1-\mathcal{A}\left(\varepsilon_{+}\right) \mathcal{A}^{\sigma}\left(\varepsilon_{-}\right)+\sqrt{\mathcal{T}\left(\varepsilon_{+}\right) \mathcal{T}\left(\varepsilon_{-}\right)} \sin ^{2}\left(\varphi_{\sigma}\right)$ with $\varepsilon_{ \pm}=\varepsilon \pm \hbar \omega_{0} / 2$, the Fermi distribution function $f_{0}(\varepsilon)$ $=\left(e^{(\varepsilon-\mu) / k_{B} T}+1\right)^{-1}$, and the Bose occupation factor $N_{T}=\left(e^{\hbar \omega_{0} / k_{B} T}-1\right)^{-1}$, both evaluated at equilibrium. Here, $\tilde{\Gamma}(\varepsilon)=\left(\Gamma_{L}(\varepsilon)+\Gamma_{R}(\varepsilon)\right) /(1+\xi(\varepsilon))$ is the (renormalized) total resonance width, where $\Gamma_{\ell}(\varepsilon)=\pi \rho_{\ell}(\varepsilon) V_{\ell}^{2}$ and $\xi(\varepsilon)=\pi^{2} \rho_{L}$ $(\varepsilon) \rho_{R}(\varepsilon) W^{2}$ is the direct tunneling coupling, with $\rho_{\ell}(\varepsilon)$ the $l$-lead density of states. The retarded Green's function in the absence of e-ph coupling is given by $\mathscr{G}_{\sigma, \sigma}^{r}(\varepsilon)=\left(\varepsilon-\varepsilon_{d}+i \tilde{\Gamma}\right.$ $\left.+\tilde{\Gamma} \sqrt{\alpha \xi} \cos \varphi_{\sigma}\right)^{-1}$ with $\alpha(\varepsilon)=4 \Gamma_{L} \Gamma_{R} /\left(\Gamma_{L}^{\sigma, \sigma}+\Gamma_{R}\right)^{2}$. We have defined the functions $\mathcal{A}(\varepsilon)=\left[\left(\Gamma_{L}-\Gamma_{R}\right) /\left(\Gamma_{L}+\Gamma_{R}\right)\right][(1-\xi) /$ $(1+\xi)]$ and $\mathcal{T}(\varepsilon)=4 \alpha \xi /(1+\xi)^{2}$. The thermopower inelastic term can be cast in the form $L_{P}\left(\varphi_{\sigma}\right)=\left(2 \lambda^{2} e / h\right) \int d \varepsilon B\left(\varepsilon, \varphi_{\sigma}\right)$ $\left[(\varepsilon-\mu) M_{0}\left(\varepsilon, \varphi_{\sigma}\right)+\left(\hbar \omega_{0} / 2\right) M_{1}\left(\varepsilon, \varphi_{\sigma}\right)\right]$, where $M_{1}\left(\varepsilon, \varphi_{\sigma}\right)$ $\equiv\left[\mathcal{A}\left(\varepsilon_{+}\right) \sqrt{\mathcal{T}\left(\varepsilon_{-}\right)}-\mathcal{A}\left(\varepsilon_{-}\right) \sqrt{\mathcal{T}\left(\varepsilon_{+}\right)}\right] \sin \varphi_{\sigma}$. Finally, the phonon assisted current contribution is $X_{P}\left(\varphi_{\sigma}\right)=\left(2 \lambda^{2} e / h\right)$ $\hbar \omega_{0} \int d \varepsilon B\left(\varepsilon, \varphi_{\sigma}\right) M_{2}\left(\varepsilon, \varphi_{\sigma}\right)$ with $M_{2}\left(\varepsilon, \varphi_{\sigma}\right) \equiv\left[\mathcal{A}\left(\varepsilon_{-}\right)-\mathcal{A}\left(\varepsilon_{+}\right)\right]$ $+\left[\sqrt{\mathcal{T}\left(\varepsilon_{+}\right)}-\sqrt{\mathcal{T}\left(\varepsilon_{-}\right)}\right] \sin \varphi_{\sigma}$.

In the wide-band limit, $\rho_{\ell}$ is energy independent. Then, $\mathcal{T}(\varepsilon)$ and $\mathcal{A}(\varepsilon)$ become constants and we find $X_{P}\left(\varphi_{\sigma}\right)=0$, i.e., the spin current $I_{\sigma}$ is insensitive to changes in $T_{P}$. Interestingly, for the general case of energy-dependent densities of states our model predicts, quite generally, a spindependent flow generated by nonzero $\Delta T_{P}$. To see this, let us analyze the charge $I_{c}=I_{\uparrow}+I_{\downarrow}$ current,

$$
\begin{aligned}
I_{c}= & {\left[G\left(\varphi_{\uparrow}\right)+G\left(\varphi_{\downarrow}\right)\right] \Delta V+\left[G\left(\varphi_{\uparrow}\right)-G\left(\varphi_{\downarrow}\right)\right] \Delta V_{s} } \\
& +\left[L\left(\varphi_{\uparrow}\right)+L\left(\varphi_{\downarrow}\right)\right] \frac{\Delta T}{T}+\left[X_{P}\left(\varphi_{\uparrow}\right)+X_{P}\left(\varphi_{\downarrow}\right)\right] \frac{\Delta T_{P}}{T},
\end{aligned}
$$

and the $\operatorname{spin} I_{s}=I_{\uparrow}-I_{\downarrow}$ current,

$$
\begin{aligned}
I_{s}= & {\left[G\left(\varphi_{\uparrow}\right)-G\left(\varphi_{\downarrow}\right)\right] \Delta V+\left[G\left(\varphi_{\uparrow}\right)+G\left(\varphi_{\downarrow}\right)\right] \Delta V_{s} } \\
& +\left[L\left(\varphi_{\uparrow}\right)-L\left(\varphi_{\downarrow}\right)\right] \frac{\Delta T}{T}+\left[X_{P}\left(\varphi_{\uparrow}\right)-X_{P}\left(\varphi_{\downarrow}\right)\right] \frac{\Delta T_{P}}{T} .
\end{aligned}
$$

In order to examine the possibility of generating pure spin current, i.e., $I_{C}=0$ and $I_{s} \neq 0$, only via local heating $\left(\Delta T_{P} \neq 0\right)$ we set $\Delta T=0$ henceforth. Due to symmetry considerations, we have $G\left(\varphi_{\uparrow}\right)=G\left(\varphi_{\downarrow}\right) \equiv G(\varphi)$. In addition, for symmetric couplings $\left(\Gamma_{L}=\Gamma_{R}\right)$ we find $X_{P}\left(\varphi_{\uparrow}\right)=-X_{P}\left(\varphi_{\downarrow}\right) \equiv X_{P}(\varphi)$. Hence, Eqs. (2) and (3) reduce to

$$
\begin{gathered}
I_{c}=2 G(\varphi) \Delta V \\
I_{s}=2 G(\varphi) \Delta V_{s}+2 X_{P}(\varphi) \frac{\Delta T_{P}}{T} .
\end{gathered}
$$

This particularly simple result is central to our proposal. When no dc voltage is applied $(\Delta V=0)$, the charge current $I_{c}$ vanishes and just a pure spin current remains. Furthermore, when the spin relaxation time in the reservoirs is very short, ${ }^{19}$ we can neglect the term proportional to the spin bias $\Delta V_{s}$ and thus $I_{s}$ is uniquely determined by the temperature difference between the phonon bath and the system. In general, our thermomagnetic device produces spinpolarized currents from temperature differences only.

For realistic systems, lead couplings can be asymmetric and we then obtain nonvanishing charge currents. However, this finite charge current can be cancelled by applying a compensating bias voltage across the system. A similar compensation effect has been proposed for a two-terminal quantum dot system. ${ }^{20}$ The difference is that we heat up the molecule using local phonon coupling and allow for (Rashba) electric fields while Ref. 20 uses Zeeman magnetic fields. Thus, our proposed device is an all-electrical setup.
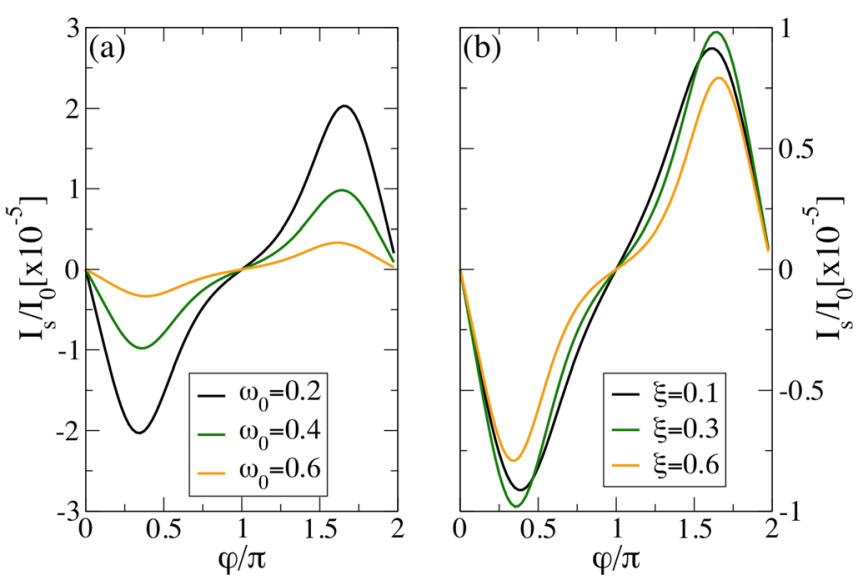

FIG. 2. Normalized spin current versus the Rashba phase $\varphi$ for (a) different phonon frequencies $\omega_{0}$ at fixed direct tunnel coupling $\xi=0.4$ and (b) different values of $\xi$ for $\omega_{0}=0.4$. Parameters: $\lambda=0.8, T=0.1, \Delta T_{P}=0.05$, $E_{d}=0.8$, and bandwidth $D=10$ with a semielliptic density of states $\rho(\varepsilon)=\sqrt{1-4(\varepsilon / D)^{2}}$. Here, energies are given in units of $\Gamma$ and the current unit is $I_{0}=e \omega_{0} \lambda^{2} / \Gamma^{2}$. 

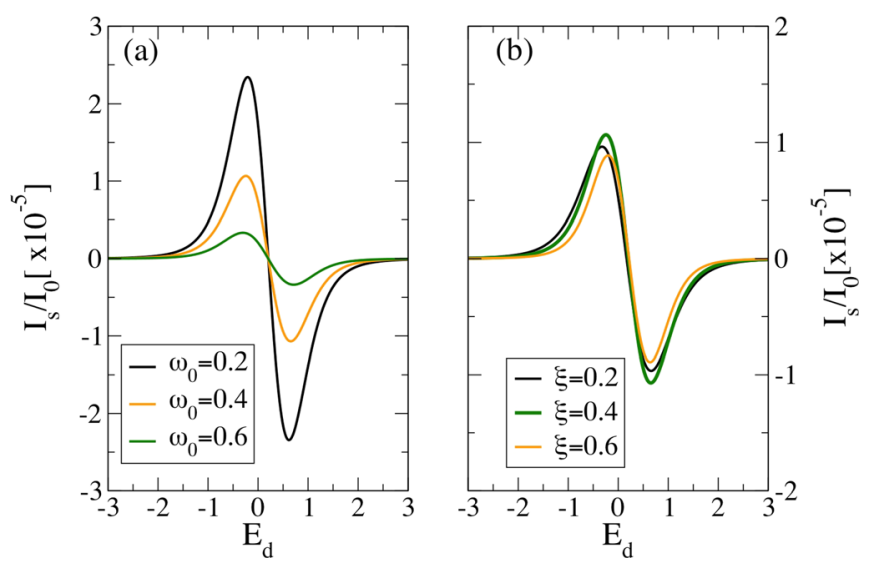

FIG. 3. Normalized spin current versus the energy level position $E_{d}$ for (a) different phonon frequency $\omega_{0}$ at fixed direct tunnel coupling $\xi=0.4$ and (b) different values of $\xi$ for $\omega_{0}=0.4$. Parameters are taken as in Fig. 2 with a fixed Rashba phase $\varphi=0.35 \pi$ near which the maximum spin current amplitude occurs.

Figures 2 and 3 display the spin currents as a function of the Rashba phase $\varphi$ and the molecular energy level $E_{d}$ for various values of the phonon frequency $\omega_{0}$ and direct tunneling strength $\xi$. Figure 2 shows oscillations of the spin current when $\varphi$ changes from 0 to $2 \pi$. At fixed temperature, the number of phonons decreases when $\omega_{0}$ grows, yielding lower spin currents as shown in Fig. 2(a). In Fig. 2(b), we observe a weak dependence of the spin current amplitude on $\xi$. This is a nice property-the generated spin polarization is robust against unintentional variations of the background transmission. Figure 3 illustrates the gate dependence of $I_{s}$ at a fixed Rashba phase. We also find that the current amplitude enhances as $\omega_{0}$ decreases. Spin currents are maximized for specific positions of the molecular level: $E_{d}=-0.25 \Gamma$ and $E_{d}=0.75 \Gamma$. Moreover, the level positions at which $I_{s}$ is maximal depends weakly on $\omega_{0}$. Finally, Fig. 3(b) presents small variation of the spin current when $\xi$ varies substantially, in agreement with the robustness discussed above.

In prototypical molecular transistors, vibrational frequencies are found to be of the order of $\mathrm{THz}$ for $C_{60}$ (Ref. 21) and carbon nanotubes. ${ }^{22}$ These systems are most suitable to test our predictions due to their intrinsic spin-orbit coupling. ${ }^{23} \mathrm{We}$ estimate from Figs. 2 and 3 that the achievable spin currents are of the order of $I_{s} \sim 1 \mathrm{pA}$ for $\Gamma=\lambda=1 \mathrm{meV}, \omega_{0}=$ $1 \mathrm{THz}, T=1 \mathrm{~K}$, and $\Delta T_{P}=0.5 \mathrm{~K}$. A similar setup also yields currents of the order of $1 \mathrm{pA} .^{5}$ This current range is small but can be detected within present techniques. ${ }^{24}$

In summary, we have proposed a spin current generator by locally heating a molecule embedded in a spin-orbit interferometer. Our device works even in the absence of voltage and temperature bias applied to the electronic terminals. Importantly, our heat-to-spin current mechanism is independent of magnetic couplings to external reservoirs, in contrast to recent proposals which emphasize interactions with ferromagnetic leads. ${ }^{25}$ We have found that optimal spin polarization can be determined by adjusting the Rashba spinorbit coupling and the energy level of the molecule. Our results are relevant in view of recent developments in the field of spin caloritronics. Analogous setups could be envisaged where spin is replaced with orbital (pseudospin) degrees of freedom. ${ }^{26}$

The authors acknowledge the support from MECD under Grant Nos. FIS2011-23526 and CSD2007-00042 (CPAN), and the National Research Foundation (NRF) grant funded by the Korea government (MSIP) (No. 20110030046).

${ }^{1}$ K. Uchida, S. Takahashi, K. Harii, J. Ieda, W. Koshibae, K. Ando, S. Maekawa, and E. Saitoh, Nature 455, 778 (2008).

${ }^{2}$ A. Slachter, F. L. Bakker, J.-P. Adam, and B. J. van Wees, Nat. Phys. 6, 879 (2010).

${ }^{3}$ G. E. W. Bauer, A. H. MacDonald, and S. Maekawa, Solid State Commun. 150, 459 (2010).

${ }^{4}$ O. Entin-Wohlman, Y. Imry, and A. Aharony, Phys. Rev. B 82, 115314 (2010).

${ }^{5}$ R. Sánchez and M. Büttiker, Phys. Rev. B 83, 085428 (2011).

${ }^{6}$ O. Entin-Wohlman and A. Aharony, Phys. Rev. B 85, 085401 (2012).

${ }^{7}$ B. Sothmann, R. Sánchez, A. N. Jordan, and M. Büttiker, Phys. Rev. B 85, 205301 (2012).

${ }^{8}$ L. Simine and D. Segal, Phys. Chem. Chem. Phys. 14, 13820 (2012).

${ }^{9}$ R. Sánchez, R. López, D. Sánchez, and M. Büttiker, Phys. Rev. Lett. 104, 076801 (2010).

${ }^{10}$ Q.-f. Sun and X. C. Xie, Phys. Rev. B 73, 235301 (2006).

${ }^{11}$ F. Chi and J. Zheng, Appl. Phys. Lett. 92, 062106 (2008).

${ }^{12}$ H.-F. Lü and Y. Guo, Appl. Phys. Lett. 91, 092128 (2007).

${ }^{13}$ E. Vernek, N. Sandler, and S. E. Ulloa, Phys. Rev. B 80, 041302(R) (2009).

${ }^{14}$ J. S. Lim, M. Crisan, D. Sánchez, R. López, and I. Grosu, Phys. Rev. B 81, 235309 (2010).

${ }^{15}$ R. Swirkowicz, M. Wierzbicki, and J. Barnas, Phys. Rev. B 80, 195409 (2009).

${ }^{16}$ T. Frederiksen, M. Brandbyge, N. Lorente, and A.-P. Jauho, Phys. Rev. Lett. 93, 256601 (2004).

${ }^{17}$ M. Galperin, A. Nitzan, and M. A. Ratner, Phys. Rev. B 75, 155312 (2007).

${ }^{18}$ H. J. W. Haug and A.-P. Jauho, Quantum Kinetics in Transport and Optics of Semiconductors (Springer, Berlin, 2008).

${ }^{19}$ M. Johnson and R. H. Silsbee, Phys. Rev. Lett. 55, 1790 (1985).

${ }^{20}$ T. Rejec, R. Zitko, J. Mravlje, and A. Ramsak, Phys. Rev. B 85, 085117 (2012).

${ }^{21}$ H. Park, J. Park, A. K. L. Lim, E. H. Anderson, A. P. Alivisatos, and P. L. McEuen, Nature 407, 57 (2000).

${ }^{22}$ R. Leturcq, Ch. Stampfer, K. Inderbitzin, L. Durrer, Ch. Hierold, E. Mariani, M. G. Schultz, F. Von Oppen, and K. Ensslin, Nat. Phys. 5, 327 (2009).

${ }^{23}$ E. D. Minot, Y. Yaish, V. Sazonova, and P. L. McEuen, Nature 428, 536 (2004).

${ }^{24}$ A. Javey, J. Guo, Q. Wang, M. Lundstrom, and H. Dai, Nature 424, 654 (2003).

${ }^{25}$ B. Sothmann and M. Büttiker, Europhys. Lett. 99, 27001 (2012).

${ }^{26}$ J. S. Lim, R. López, and D. Sánchez, e-print arXiv:1306.1726. 